\section{Retrospectiva de 20 anos de atividade da Unidade de Farmacovigilância do Porto, Portugal}

\author{
Looking back on 20 years of work at the \\ Porto Pharmacovigilance Centre, Portugal
}

\section{Retrospectiva de 20 años de actividad de la Unidad de Farmacovigilancia de Oporto, Portugal}

\author{
Renato Ferreira-da-Silva 1,2,3 \\ Inês Ribeiro-Vaz 1,2,3 \\ Ana Marta Silva 1,2,3 \\ Joana Marques 1,2,3 \\ Jorge Junqueira Polónia 1,2,3
}

doi: 10.1590/0102-311X00304420

\section{Resumo}

Desde 1963 que a farmacovigilância foi reconhecida como uma área prioritária na saúde pública a nível global pela Organização Mundial da Saúde, garantindo a monitorização permanente da segurança dos medicamentos. $O$ objetivo deste trabalho foi caracterizar as reações adversas a medicamentos recebidas pela Unidade de Farmacovigilância do Porto (UFPorto), Portugal, ao longo de duas décadas de atividade. Foram consideradas todas as notificações de suspeitas de reações adversas a medicamentos recebidas entre janeiro de 2001 e dezembro de 2019. Foram calculadas as taxas de notificação anuais, assim como a sua distribuição por origem, tipo de notificador e local de exercício da atividade, gravidade, conhecimento prévio e causalidade das reações adversas a medicamentos notificadas. No período em estudo, a UFPorto recebeu 9.711 notificações de suspeitas de reações adversas a medicamentos. As instituições hospitalares são aquelas que mais notificam $(n=6.003$; $64 \%$ ), assim como o médico entre os profissionais de saúde ( $n=5.284 ; 54,4 \%)$. Os eventos adversos mais frequentemente reportados são graves $(n=6.275$; 72\%) e encontram-se descritos no respectivo Resumo das Características do Medicamento $(n=6.978 ; 72 \%)$. À maioria das notificações avaliadas pela UFPorto foi atribuído o grau de causalidade "provável" ( $n=7.473 ; 77 \%)$, independentemente do tipo de notificador. Os resultados obtidos são concordantes com outros dados previamente reportados na literatura médica internacional e em relatórios oficiais nacionais. Contudo, continua-se a verificar taxas de subnotificação acentuadas, face ao esperado. Ao longo de aproximadamente 20 anos de atividade da UFPorto, tem-se verificado um aumento da sua atividade nas diversas vertentes da segurança do medicamento.

Farmacovigilância; Efeitos Colaterais e Reações Adversas Relacionados a Medicamentos; Sistemas de Notificação de Reações Adversas a Medicamentos; Monitoramento de Medicamentos

\author{
Correspondência \\ R. Ferreira-da-Silva \\ Unidade de Farmacovigilância do Porto, Infarmed, I.P./ \\ Faculdade de Medicina, Universidade do Porto. \\ Rua Doutor Plácido da Costa, 4200-450 Porto, Portugal. \\ renato.ivos@gmail.com \\ 1 Unidade de Farmacovigilância do Porto, Infarmed, I.P./
Universidade do Porto, Porto, Portugal.
2 Faculdade de Medicina, Universidade do Porto, Porto,
Portugal.
${ }^{3}$ CINTESIS - Centro de Investigação em Tecnologia e Serviços
de Saúde, Porto, Portugal.
}




\section{Introdução}

"The hazard may be insignificant or may be acceptable in relation to the drug's therapeutic action" (Comitê de Segurança de Medicamentos, Reino Unido, 1969/1970 1).

\section{Aspectos históricos e evolução do conceito de Farmacovigilância}

De acordo com a definição de 1972 da Organização Mundial da Saúde (OMS), as reações adversas a medicamentos podem ser definidas como respostas prejudiciais e indesejadas a um medicamento que ocorrem em doses habitualmente usadas para profilaxia, diagnóstico ou tratamento, e às quais se atribui um nexo de causalidade entre a ocorrência adversa e o medicamento ${ }^{2}$. No entanto esta definição de reações adversas a medicamentos foi alterada a nível europeu pela Diretiva 2010/84/EU do Parlamento Europeu e do Conselho de 15 de dezembro, pelo que desde 2012 se define como "uma reação nociva e não intencional a um medicamento", passando a incluir a utilização dessa medicação fora dos pressupostos da sua Autorização de Introdução no Mercado (AIM), tais como o abuso, exposição ocupacional ou utilização indevida ${ }^{3}$. A ideia de que todos os medicamentos têm implícita a possibilidade de induzir efeitos adversos (ou reações adversas a medicamentos) parece estar bem estabelecida entre a comunidade clínica e de usuários, pelo que a decisão terapêutica terá sempre de ponderar os "benefícios" esperados do medicamento, assim como os "riscos" da sua utilização. Da relação destes dois termos resulta um conceito mais robusto - "segurança" do medicamento - pelo que o peso dado a cada uma destas definições é relativo e variável entre patologias e doentes 4 .

O pressuposto de que os medicamentos não são desprovidos de riscos já remete para muitas décadas atrás. Se o desastre da talidomida marcou o caminho que se viria a fazer na farmacovigilância e segurança dos medicamentos anos mais tarde, já em 1937, com o início da "era sulfamídica" ficou claro que a morte de 107 indivíduos por insuficiência renal pela utilização do dietilenoglicol como solvente do elixir da sulfanilamida viria a traduzir-se em mais legislação 5. Em 1938, é publicado o primeiro grande documento que leva os produtores de medicamentos a comprovar a segurança dessas novas substâncias. A Lei Federal de Alimentos, Medicamentos e Cosméticos dos Estados Unidos (Federal Food, Drug and Cosmetic Act) remete para as primeiras obrigatoriedades no âmbito da toxicologia experimental/pré-clínica, equiparando-se à atual fase I dos ensaios clínicos 6 . Mais tarde, nos anos 1950, o conceito de "reação adversa" começa a marcar a terminologia clínica com o surgimento de casos de anemia aplástica pela utilização do antibiótico cloranfenicol, hoje com efeitos adversos bem caracterizados como a depressão medular 7,8 . Se estes dois eventos da história do medicamento são relembrados sempre que se fala em farmacovigilância e segurança dos medicamentos, o "desastre ou drama da talidomida" marcou de forma muito significativa a era da regulamentação de medicamentos pós-comercialização, isto é, após a atribuição de AIM pelas autoridades reguladoras que, no caso de Portugal, é a Autoridade Nacional do Medicamento e Produtos de Saúde (Infarmed, I.P.), e no contexto europeu é a Agência Europeia de Medicamentos (EMA).

A 16 de dezembro de 1961, a prestigiada revista The Lancet publicou uma importante carta ao editor redigida por William McBride 9 , um obstetra australiano, dando conta da tragédia de 10 mil crianças com focomelia por exposição intrauterina à talidomida. Este fármaco, à data utilizado como hipnótico suave e profilático dos enjoos matinais da gravidez, foi utilizado durante vários anos na prática clínica como um fármaco "mágico" para as grávidas. Atualmente, a talidomida é ainda utilizada na prática clínica como primeira linha (em combinação com outros fármacos) para doentes $\geq 65$ anos, com mieloma múltiplo não tratado ou não elegíveis para tratamento com altas doses de quimioterapia 10. Sendo um dos fármacos mais importantes do século $\mathrm{XX}$, a talidomida mobilizou a legislação e a regulamentação dos medicamentos, surgindo os primeiros conceitos de farmacovigilância e segurança das substâncias destinada à profilaxia, ao diagnóstico ou a tratamentos médicos. Atualmente, a utilização da talidomida segue um rigoroso plano de gestão do risco que inclui um apertado plano de prevenção da gravidez. No seguimento deste flagelo de saúde pública, desencadeou-se uma cascata de iniciativas legislativas nos Estados Unidos, iniciando-se em 1962 com a Emenda à Lei Federal de Alimentos, Medicamentos e Cosméticos de 1938, fazendo referência à importância da investigação clínica na monitorização e estudo dos efeitos iatrogênicos dos medicamentos 5 . 
A partir da década de 1960, um conjunto de desafios foi assumido pelas autoridades de saúde a nível mundial, resultando em novas diretrizes para garantia da proteção da saúde pública 11 .

\section{A farmacovigilância em Portugal e a evolução da Unidade de Farmacovigilância do Porto}

$\mathrm{Na}$ União Europeia existe uma rede de regulamentação composta pelas autoridades competentes dos Estados-membro, a Comissão Europeia e a EMA, que é responsável pela concessão de AIM e pela supervisão dos medicamentos, na qual se inclui a farmacovigilância. Em 2012 entrou em vigor uma nova legislação que visa essencialmente reforçar o papel da farmacovigilância durante o ciclo de vida do medicamento, caracterizada pelo aumento da transparência do sistema, recomendando a criação de portais nacionais.

Em Portugal, a evolução do número de notificações de reações adversas a medicamentos no Sistema Nacional de Farmacovigilância (SNF) de 1992 a 2017 tem refletido um aumento constante e sustentado. O médico continua a ser o profissional de saúde que mais notifica, sendo as especialidades médicas de Imunoalergologia e Reumatologia as que mais se destacam por força dos protocolos celebrados com as unidades regionais de farmacovigilância. Também o farmacêutico hospitalar apresenta um aumento significativo, com o triplo das notificações do farmacêutico comunitário em 2017. De uma forma global, cerca de três quartos dos casos notificados são graves, e a classificação de órgãos e sistemas do dicionário MedDRA (https://www.meddra.org/how-to-use/support-docu mentation/portuguese) define as perturbações gerais e alterações no local de administração (geral) com mais notificações, seguida da pele e dos tecidos subcutâneos. Apesar de o SNF de Portugal ter sido iniciado há 25 anos, observa-se um desenvolvimento assinalável, resultado do investimento que tem sido realizado tanto na divulgação como na implementação de ferramentas tecnológicas de apoio à monitorização 3 .

A Unidade de Farmacovigilância do Porto (UFPorto), uma das unidades regionais do SNF de Portugal, resultou da articulação institucional entre unidades prestadoras de cuidados de saúde, as instituições acadêmicas e o Infarmed. A UFPorto, à semelhança das restantes nove unidades de farmacovigilância existentes em território nacional, assume como seu principal objetivo a divulgação e a promoção da notificação de reações adversas a medicamentos entre os profissionais de saúde e dos usuários do distrito do Porto, criando uma proximidade com as instituições de saúde. Em 2000, aquando da inauguração da unidade, recebeu a designação de Unidade de Farmacovigilância do Norte (UFN), que manteve até ao final de 2016, altura em que se passou a designar por UFPorto, como resultado da estratégia de descentralização implementada no SNF. Ao longo dos 20 anos de atividade, a UFPorto tem vindo a salientar-se no plano nacional pela implementação de vários sistemas de informação de apoio à sua atividade, tendo sido, por exemplo, a primeira entidade nacional a criar um formulário de notificação online de reações adversas a medicamentos, em 2005. A UFPorto está sediada na Faculdade de Medicina da Universidade do Porto. Desde janeiro de 2017, a UFPorto está adstrita à região do distrito do Porto, abrangendo 1,8 milhão de habitantes e 24 mil profissionais de saúde exercendo atividade em 281 instituições de saúde pertencentes ao Serviço Nacional de Saúde e cerca de 430 farmácias comunitárias.

Todas as notificações recebidas e validadas na UFPorto são alvo de codificação e introdução na base de dados do Infarmed (Portal de Notificação de Reações Adversas a Medicamentos - Portal RAM. https://www.infarmed.pt/web/infarmed/portalram), sendo enviadas diretamente para a base de dados europeia - EudraVigilance (https://www.ema.europa.eu/en/human-regulatory/researchdevelopment/pharmacovigilance/eudravigilance). Posteriormente, cada caso é avaliado de forma individual, sendo efetuada uma avaliação de causalidade (ou atribuição de grau de probabilidade, de acordo com os critérios Centro de Monitoramento de Uppsala da Organização Mundial da Saúde - WHO-UMC Causality Categories 12 ). Após a avaliação por parte da Unidade, cada notificador (profissional de saúde ou usuário) recebe feedback, dando conta do grau de probabilidade atribuído à suspeita de reações adversas a medicamentos. Este feedback é grandemente valorizado pelos notificadores, sendo a única forma de retribuição imediata pela colaboração prestada ao SNF, aumentando assim o envolvimento da sociedade com a farmacovigilância.

O objetivo deste trabalho é caracterizar as notificações de suspeitas de reações adversas a medicamentos recebidas na UFPorto quanto a sua origem, tipo de notificador, local do exercício da atividade dos notificadores, gravidade, conhecimento prévio e causalidade atribuída. 


\section{Metodologia}

Este estudo, observacional, retrospetivo e descritivo, considerou todas as notificações de suspeitas de reações adversas a medicamentos que foram reportadas à UFPorto desde janeiro de 2001 e dezembro de 2019. Foram incluídas as notificações que verificaram os critérios mínimos de validação, designadamente: doente, notificador, medicamento(s) suspeito(s) e evento(s) adverso(s).

Os dados foram obtidos a partir da base de dados do Infarmed (Portal RAM), tendo sido incluídas todas as reações adversas a medicamentos inseridas no Portal no período indicado, independentemente do grau de causalidade atribuído após a sua avaliação.

As notificações das suspeitas de reações adversas a medicamentos são descritas de acordo com sua relevância, nomeadamente, a gravidade (grave ou não grave), grau de probabilidade conforme a escala da OMS (provável, possível, definitiva, condicional, não classificável, improvável ou não aplicável) 12 e o conhecimento prévio da reações adversas a medicamentos (descrita ou não descrita no Resumo das Características do Medicamento - RCM). As reações adversas a medicamentos foram consideradas graves se "resultaram em morte", "colocaram a vida em risco", "motivaram ou prolongaram a hospitalização", "motivaram incapacidade temporária e/ou definitiva", e/ou "originaram anomalias congênitas". Outros eventos adversos, que apesar de à data da sua ocorrência não terem colocado a vida em risco ou motivado a hospitalização do usuário, mas que estiveram associados a condições clínicas importantes e/ou exigiram a intervenção médica, foram classificadas como graves 13 . O grau de probabilidade foi atribuído pelo perito clínico da UFPorto de acordo com o método de introspeção global, avaliando-se a relação causal entre a exposição a determinado medicamento (ou vários) e a suspeita do(s) evento(s) adverso(s), tendo em conta o algoritmo de Karsh \& Lasagna 14. As reações adversas a medicamentos foram classificadas como descritas se constassem do respetivo RCM.

Os dados relativos aos doentes são anônimos e nenhuma informação confidencial relativa ao processo clínico dos usuários foi incluída na presente análise descritiva. O tratamento dos dados cumpre os requisitos legais e europeus em matéria de proteção de dados, garantindo-se a sua segurança e confidencialidade, sendo que essa informação não é partilhada com entidades externas ao SNF.

Para o tratamento estatístico dos dados, foi o utilizado o software Microsoft 365 Excel (https:// products.office.com/).

\section{Resultados}

No período analisado, foram registadas 9.711 notificações de suspeitas de reações adversas a medicamentos no Portal do Infarmed, relativas à unidade de farmacovigilância referida. A consulta das tabelas permite conhecer os números relativos às notificações processadas ao longo de aproximadamente 20 anos de existência da UFPorto (Tabela 1) e a sua respectiva distribuição por tipo de instituição (Tabela 2).

Ao longo dos anos, embora com ligeiras variações, o número de notificações de suspeitas de reações adversas a medicamentos tem aumentado, tendo-se atingindo o número mais alto de notificações de suspeitas de reações adversas a medicamentos em $2015(\mathrm{n}=1.042)$. A partir de 2017, quando a área adstrita à UFPorto passou a ser apenas o distrito do Porto, foram processadas 685 e 749 notificações/ ano, correspondendo aos anos de 2017 e 2018, respectivamente. Em 2019, a UFPorto recebeu 906 notificações de suspeitas de reações adversas a medicamentos. As porcentagens de reações adversas a medicamentos graves e não descritas no RCM, que são aquelas que motivam maior preocupação de análise, não sofreram variações importantes nos anos subsequentes. Embora não incluído na Tabela 1, no decorrer do primeiro semestre de 2020 foram notificadas 234 suspeitas de reações adversas a medicamentos.

As suspeitas de reações adversas mais frequentemente reportadas estão classificadas como graves ( $\mathrm{n}=6.275 ; 65 \%)$ e descritas no RCM do medicamento $(\mathrm{n}=6.978 ; 72 \%)$. Observa-se também uma tendência crescente do número de reações adversas a medicamentos não descritas no RCM até 2016, com diminuição nos últimos três anos analisados. 
Tabela 1

Evolução do número de notificações ao longo dos anos, por critério de gravidade (grave/não grave) e por conhecimento prévio da reação adversa a medicamentos (descrita/não descrita).

\begin{tabular}{|c|c|c|c|c|c|c|c|c|c|}
\hline \multirow[t]{2}{*}{ Unidade/Ano } & \multirow{2}{*}{$\begin{array}{c}\text { Notificações } \\
n\end{array}$} & \multicolumn{2}{|c|}{ Graves } & \multicolumn{2}{|c|}{ Não graves } & \multicolumn{2}{|c|}{ Descritas no RCM } & \multicolumn{2}{|c|}{ Não descritas no RCM } \\
\hline & & $\mathbf{n}$ & $\%$ & $\mathbf{n}$ & $\%$ & $\mathbf{n}$ & $\%$ & $\mathbf{n}$ & $\%$ \\
\hline \multicolumn{10}{|l|}{ UFN } \\
\hline 2001 & 145 & 62 & 43,0 & 83 & 57,0 & 128 & 88,0 & 17 & 12,0 \\
\hline 2002 & 162 & 86 & 53,0 & 76 & 47,0 & 126 & 78,0 & 36 & 22,0 \\
\hline 2003 & 132 & 61 & 46,0 & 71 & 54,0 & 107 & 81,0 & 25 & 19,0 \\
\hline 2004 & 640 & 345 & 54,0 & 295 & 46,0 & 487 & 76,0 & 153 & 24,0 \\
\hline 2005 & 362 & 205 & 57,0 & 157 & 43,0 & 276 & 76,0 & 86 & 24,0 \\
\hline 2006 & 343 & 204 & 59,0 & 139 & 41,0 & 280 & 82,0 & 63 & 18,0 \\
\hline 2007 & 315 & 174 & 55,0 & 141 & 45,0 & 259 & 82,0 & 56 & 18,0 \\
\hline 2008 & 327 & 184 & 56,0 & 143 & 44,0 & 274 & 84,0 & 53 & 16,0 \\
\hline 2009 & 331 & 175 & 53,0 & 156 & 47,0 & 242 & 73,0 & 89 & 27,0 \\
\hline 2010 & 372 & 249 & 67,0 & 123 & 33,0 & 245 & 66,0 & 127 & 34,0 \\
\hline 2011 & 411 & 284 & 69,0 & 127 & 31,0 & 258 & 63,0 & 153 & 37,0 \\
\hline 2012 & 423 & 280 & 66,0 & 143 & 34,0 & 266 & 63,0 & 157 & 37,0 \\
\hline 2013 & 480 & 339 & 71,0 & 141 & 29,0 & 269 & 56,0 & 211 & 44,0 \\
\hline 2014 & 932 & 563 & 60,0 & 369 & 40,0 & 641 & 69,0 & 291 & 31,0 \\
\hline 2015 & 1.042 & 663 & 64,0 & 379 & 36,0 & 663 & 64,0 & 379 & 36,0 \\
\hline 2016 & 954 & 634 & 66,0 & 320 & 34,0 & 630 & 66,0 & 324 & 34,0 \\
\hline \multicolumn{10}{|l|}{ UFPorto } \\
\hline 2017 & 685 & 511 & 75,0 & 174 & 25,0 & 543 & 79,0 & 142 & 21,0 \\
\hline 2018 & 749 & 552 & 74,0 & 197 & 26,0 & 572 & 76,0 & 177 & 24,0 \\
\hline 2019 & 906 & 704 & 78,0 & 202 & 22,0 & 712 & 79,0 & 194 & 21,0 \\
\hline Total & 9.711 & 6.275 & 65,0 & 3.436 & 35,0 & 6.978 & 72,0 & 2.733 & 28,0 \\
\hline
\end{tabular}

RCM: Resumo das Características do Medicamento; UFN: Unidade de Farmacovigilância do Norte; UFPorto: Unidade de Farmacovigilância do Porto.

Tabela 2

Número de notificações de profissionais de saúde por distrito, segundo o tipo de instituição do notificador, Portugal.

\begin{tabular}{|c|c|c|c|c|c|c|c|c|c|c|c|c|c|c|c|}
\hline \multirow[t]{2}{*}{ Distrito } & \multirow{2}{*}{$\begin{array}{c}\text { Notificações } \\
\text { n }\end{array}$} & \multicolumn{2}{|c|}{ Hospital } & \multicolumn{2}{|c|}{$\begin{array}{l}\text { Centro de } \\
\text { saúde }\end{array}$} & \multicolumn{2}{|c|}{$\begin{array}{c}\text { Farmácia } \\
\text { comunitária }\end{array}$} & \multicolumn{2}{|c|}{$\begin{array}{c}\text { Outro local } \\
\text { (instituição } \\
\text { de saúde) }\end{array}$} & \multicolumn{2}{|c|}{$\begin{array}{l}\text { Outro local (não } \\
\text { instituição de } \\
\text { saúde) }\end{array}$} & \multicolumn{2}{|c|}{ Desconhecido } & \multicolumn{2}{|c|}{ Não aplicável * } \\
\hline & & $\mathbf{n}$ & $\%$ & $\mathbf{n}$ & $\%$ & $\mathbf{n}$ & $\%$ & $\mathbf{n}$ & $\%$ & $\mathbf{n}$ & $\%$ & $\mathbf{n}$ & $\%$ & $\mathbf{n}$ & $\%$ \\
\hline Aveiro & 77 & 5 & 6,5 & 60 & 78,0 & 5 & 6,5 & 6 & 7,8 & 0 & 0,0 & 1 & 1,3 & 0 & 0,0 \\
\hline Braga & 1.013 & 422 & 42,0 & 342 & 34,0 & 230 & 23,0 & 15 & 1,5 & 2 & 0,2 & 1 & 0,1 & 1 & 0,1 \\
\hline Bragança & 457 & 54 & 12,0 & 83 & 18,0 & 319 & 70,0 & 0 & 0,0 & 0 & 0,0 & 1 & 0,2 & 0 & 0,0 \\
\hline Guarda & 4 & 0 & 0,0 & 0 & 0,0 & 4 & 100,0 & 0 & 0,0 & 0 & 0,0 & 0 & 0,0 & 0 & 0,0 \\
\hline Porto & 7.179 & 5.268 & 73,0 & 894 & 12,0 & 619 & 8,6 & 227 & 3,2 & 130 & 1,8 & 25 & 0,4 & 16 & 0,2 \\
\hline Viana do & 127 & 74 & 58,0 & 32 & 25,0 & 19 & 15,0 & 2 & 1,6 & 0 & 0,0 & 0 & 0,0 & 0 & 0,0 \\
\hline \multicolumn{16}{|l|}{ Castelo } \\
\hline Vila Real & 470 & 171 & 36,0 & 113 & 24,0 & 172 & 37,0 & 14 & 3,0 & 0 & 0,0 & 0 & 0,0 & 0 & 0,0 \\
\hline Viseu & 27 & 9 & 33,0 & 16 & 59,0 & 1 & 3,7 & 1 & 3,7 & 0 & 0,0 & 0 & 0,0 & 0 & 0,0 \\
\hline Desconhecido & 14 & 0 & 0,0 & 2 & 14,0 & 0 & 0,0 & 2 & 14,0 & 0 & 0,0 & 9 & 64,0 & 1 & 7,1 \\
\hline Notificações & 9.368 & 6.003 & 64,0 & 1.542 & 16,5 & 1.369 & 14,6 & 267 & 2,9 & 132 & 1,4 & 37 & 0,5 & 18 & 0,2 \\
\hline
\end{tabular}

* Notificador profissional de saúde que não exerce atividade (reformado ou desempregado). 
Da análise da Tabela 2, infere-se que as instituições hospitalares continuam a ser aquelas que mais notificam as suspeitas de reações adversas a medicamentos ( $\mathrm{n}=6.003 ; 64 \%)$. Os centros de saúde ( $\mathrm{n}=1.542 ; 16,5 \%)$ e as farmácias comunitárias ( $\mathrm{n}=1.369 ; 14,6 \%)$, enquanto unidades prestadoras de cuidados de saúde primários, apresentam também um número de notificações bastante relevante, em comparação com outras instituições (serviços de saúde ou não). Relativamente à distribuição geográfica, até ao ano de 2016, o distrito do Porto foi aquele que mais contribuiu para o aumento do número de notificações na região Norte de Portugal $(\mathrm{n}=7.179)$, mantendo um número significativo de notificações aquando da alteração da área de atuação da UFPorto.

Na Tabela 3 é apresentado o grau de imputação de causalidade (WHO-UMC Causality Categories), com uma distribuição por tipo de notificador (profissionais de saúde e usuários).

A maioria das notificações avaliadas pela UFPorto apresenta o grau de probabilidade de "provável" ( $\mathrm{n}=7.473 ; 77 \%)$, independentemente do notificador, em comparação com os restantes graus de classificação. Seguem-se os graus de "possível" ( $n=1.505 ; 15,5 \%)$, "definitiva” ( $=444 ; 4,6 \%)$ e "condicional” ( $\mathrm{n}=279 ; 2,9 \%)$ nas classificações das suspeitas de reações adversas a medicamentos, sendo que os graus "não classificável”, "improvável” e "não aplicável” foram atribuídos a um número muito reduzido das notificações recebidas.

Se a inclusão dos usuários como agentes notificadores foi uma das conquistas do SNF, os profissionais de saúde continuam a ser aqueles que mais notificam $(\mathrm{n}=9.368 ; 96,5 \%)$. No período avaliado, foram recebidas 343 notificações provenientes de usuários, correspondendo a 3,5\% do total de notificações. A Figura 1 mostra o número de notificações realizadas por profissionais de saúde e usuários ao longo dos anos.

Entre os profissionais de saúde, o médico é aquele que mais notifica as suspeitas de reações adversas a medicamentos $(\mathrm{n}=5.284 ; 54,4 \%)$, seguindo-se o farmacêutico ( $\mathrm{n}=2.790 ; 28,7 \%)$ e, por fim, o enfermeiro $(\mathrm{n}=1.205 ; 12,4 \%)$. Por meio dos dados reportados, verifica-se também um aumento, ainda que ligeiro, do número de notificações pelo usuário a partir do ano de 2013.

\section{Discussão}

Todos os medicamentos introduzidos no mercado apresentam um perfil de segurança que resulta da relação benefício-risco que é assumida para cada indivíduo em particular ${ }^{4}$. É necessário que a farmacovigilância monitorize a segurança dos medicamentos, recorrendo para isso a diferentes métodos e ferramentas 15 .

Tabela 3

Grau de probabilidade atribuído pela Unidade de Farmacovigilância do Porto (UFPorto), Porto, Portugal, por tipo de notificador.

\begin{tabular}{|c|c|c|c|c|c|c|c|c|c|c|c|c|}
\hline \multirow[t]{2}{*}{ Grau de probabilidade } & \multicolumn{2}{|c|}{ Médico } & \multicolumn{2}{|c|}{ Farmacêutico } & \multicolumn{2}{|c|}{ Enfermeiro } & \multicolumn{2}{|c|}{$\begin{array}{l}\text { Outro } \\
\text { profissional de } \\
\text { saúde }\end{array}$} & \multicolumn{2}{|c|}{ Usuário } & \multicolumn{2}{|c|}{ Total } \\
\hline & $\mathbf{n}$ & $\%$ & $\mathbf{n}$ & $\%$ & $\mathbf{n}$ & $\%$ & $\mathbf{n}$ & $\%$ & $\mathbf{n}$ & $\%$ & $\mathbf{n}$ & $\%$ \\
\hline Notificações & 5.284 & 54,4 & 2.790 & 28,7 & 1.205 & 12,4 & 89 & 0,9 & 343 & 3,5 & 9.711 & 100,0 \\
\hline Provável & 4.154 & 78,6 & 2.000 & 71,7 & 1.022 & 84,8 & 66 & 74,2 & 231 & 67,3 & 7.473 & 77,0 \\
\hline Possível & 705 & 13,3 & 582 & 20,9 & 128 & 10,6 & 12 & 13,5 & 78 & 22,7 & 1.505 & 15,5 \\
\hline Definitiva & 300 & 5,7 & 101 & 3,6 & 24 & 2,0 & 7 & 7,9 & 12 & 3,5 & 444 & 4,6 \\
\hline Condicional & 121 & 2,3 & 105 & 3,8 & 28 & 2,3 & 4 & 4,5 & 21 & 6,1 & 279 & 2,9 \\
\hline Não classificável & 0 & 0,0 & 2 & 0,1 & 0 & 0,0 & 0 & 0,0 & 1 & 0,3 & 3 & 0,0 \\
\hline Improvável & 1 & 0,0 & 0 & 0,0 & 0 & 0,0 & 0 & 0,0 & 0 & 0,0 & 1 & 0,0 \\
\hline Não aplicável & 3 & 0,1 & 0 & 0,0 & 3 & 0,2 & 0 & 0,0 & 0 & 0,0 & 6 & 0,1 \\
\hline
\end{tabular}


Figura 1

Evolução de notificações de reações adversas a medicamentos recebidas na Unidade de Farmacovigilância do Porto (UFPorto), Porto, Portugal, por tipo de notificador, entre 2001 e 2019.
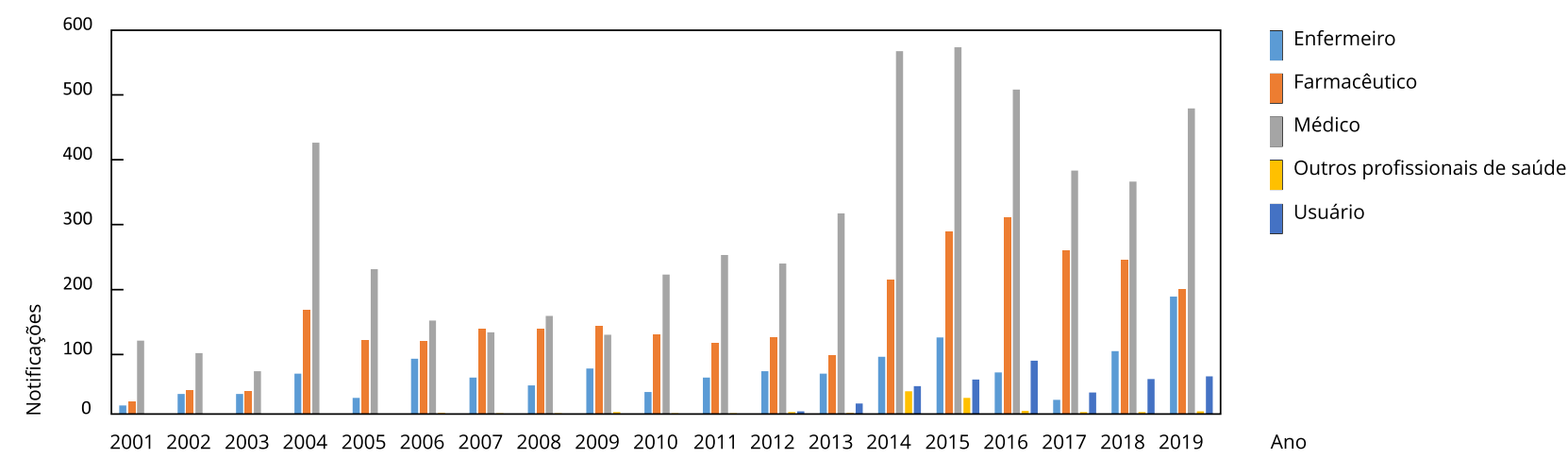

Entre os métodos mais utilizados e mais acessíveis à prática clínica dos profissionais de saúde está a notificação espontânea de reações adversas a medicamentos (farmacovigilância ativa). Em Portugal, a notificação espontânea pode ser realizada por meio do Portal RAM do Infarmed (https://www. infarmed.pt/web/infarmed/submissaoram), seja por profissionais de saúde ou usuários. Apesar de inaugurado em 2012, este portal sofreu reestruturações que culminou num novo Portal RAM em 2017. Esta nova versão permitiu agregar três grandes funcionalidades: notificação online (front office), gestão e processamentos das notificações (back-office) e o envio automatizado e centralizado de casos para a EudraVigilance, a base de dados da EMA 3. Entre os anos de 2001 e 2010, embora se tenha assistido a uma tendência de aumento do número de total de notificações, esse aumento não é regular de ano para ano, uma vez que se verificaram neste período alterações quer no número de unidades de farmacovigilância, quer nas áreas geográficas atribuídas a cada uma dessas unidades. Em 2012, assinalaram-se diversas e significativas alterações no SNF decorrentes da implementação da nova legislação europeia sobre farmacovigilância. Os usuários passam também a poder notificar e, paralelamente aos formulários em papel existentes para profissionais de saúde e para usuários, é criado o Portal RAM, tal como exposto anteriormente. No período de 2012 a 2017, as notificações de reações adversas a medicamentos inseridas diretamente no Portal RAM pelo notificador (profissionais de saúde e usuários), representam em média $13 \%$ do total de notificações, estando os restantes $87 \%$ distribuídos por outras vias (telefone, ficha de notificação ou por e-mail) 4. Desde 2013 as notificações no Portal RAM passaram a diminuir a nível nacional, tendo-se verificado uma inflexão no ano de 2017, na sequência da entrada em vigor do novo Portal RAM 3 . Embora esta reestruturação tenha permitido um melhor desempenho diante da versão anterior, marcada por maior facilidade e rapidez na notificação, a tendência nacional de diminuição de notificações online entre 2013 e 2017 não se verificou na UFPorto (na altura, designada por UFN) 4. Em 2017, o SNF sofre uma reorganização, tendo sido criadas novas unidades regionais de farmacovigilância, que passam de quatro para sete e, mais tarde, em 2018, foi criada a unidade dos Açores. Assim, é esperado um incremento assinalável no número de notificações de reações adversas a medicamentos em paralelo com o aumento do número de ações de formação em farmacovigilância realizadas em locais mais próximos e acessíveis para os notificadores. A partir de 2014, verificou-se uma tendência crescente no número de notificações anual (com um pico em 2015, com 1.042 notificações), fortemente influenciada pelo reforço de recursos humanos na UFPorto, permitindo maior integração de registos eletrônicos da base de dados interna, em simultâneo com uma maior atividade de promoção de reações adversas a medicamentos junto dos profissionais e dos usuários. Também em 2017, a área de atuação da UFPorto se alterou, pelo que se torna inviável uma 
análise rigorosa sobre os dados reportados a partir dessa data, em comparação com os anos anteriores, dificultando a interpretação sobre o impacto do novo Portal RAM na região Norte de Portugal. As 234 notificações reportadas no decorrer do primeiro semestre de 2020 refletem uma taxa de subnotificação preocupante, fortemente influenciada pela atual situação de pandemia por COVID-19. No entanto, em 2021, tem-se verificado um crescimento bastante significativo em comparação com o primeiro semestre de 2020, com a recessão de 2348 suspeitas de reações adversas a medicamentos, das quais 2.121 são relativas às vacinas contra a COVID-19. A UFPorto estabeleceu protocolos de colaboração com diversos hospitais do distrito do Porto, estando a monitorar reações adversas a medicamentos em doentes COVID-19 (em regime de internação), mas também a todos os usuários que estão a ser imunizados com as diferentes vacinas contra a COVID-19 que vão sendo aprovadas e disponibilizadas no mercado ao longo do tempo.

A carga de trabalho a que os profissionais de saúde estão sujeitos na atual situação de saúde pública, a preocupação crescente com a eficácia dos medicamentos, assim como a forte diminuição das atividades de promoção e sensibilização de reações adversas a medicamentos podem estar entre os fatores considerados para justificar a subnotificação observada no decorrer do ano de 2020. Em 2021, e apesar da carga que continua a ser sentida nos serviços de saúde, prevê-se um aumento do número de notificações, fortemente potenciado pelos programas de monitorização ativa que estão sendo implementados pelas unidades de farmacovigilância.

Apesar do usuário ser parte integrante do SNF, o contributo em termos de notificação espontânea tem sido residual 16. No entanto, a literatura sugere que a perspectiva oferecida no que diz respeito à reações adversas a medicamentos notificada por um profissional de saúde ou por usuário é bastante diferente. Os primeiros focam-se na causalidade entre o medicamento suspeito e a reação adversa, enquanto que os usuários valorizam sobretudo a gravidade e o impacto na qualidade de vida 17.

Mais de metade dos casos (65\%) reportados à UFPorto dizem respeito a reações adversas graves a medicamentos. Este resultado é consistente com as taxas de iatrogenia grave identificadas noutras unidades de farmacovigilância nacionais em anos anteriores 4,16,18. Embora a evidência seja limitada, parece que os profissionais de saúde estão particularmente sensibilizados para a notificação de reações adversas graves a medicamentos, uma vez que estas estão fortemente associadas a aumento da morbilidade e dos custos em saúde ${ }^{19}$. Nos Estados Unidos, os resultados negativos associados às reações adversas a medicamentos verificam-se, desde logo, nos elevados índices de morbilidade e mortalidade, traduzindo-se na quarta a sexta causa de morte no país ${ }^{20}$. Na União Europeia, as reações adversas a medicamentos representam 6,5\% das hospitalizações e 197 mil mortes por ano 21. Torna-se, então, fundamental garantir a cooperação de todo o sistema de saúde na identificação e notificação das reações adversas a medicamentos. As reações adversas a medicamentos traduzem-se num grave problema de saúde pública a nível global, sendo consensual a causalidade atribuída entre a sua ocorrência e os resultados negativos em saúde. As interações medicamentosas, a idade e os erros de administração têm sido apontados como as causas mais prováveis na ocorrência de efeitos adversos 22,23. Embora seu impacto seja transversal a toda a comunidade, os resultados negativos decorrentes de reações adversas a medicamentos apresentam maior gravidade nos grupos populacionais mais vulneráveis, tal como nos pacientes idosos com patologia do foro cognitivo 24. A subnotificação de reações adversas a medicamentos nessa população é atribuída aos sinais e sintomas inespecíficos e, sobretudo, ao viés de memória prevalente entre estes indivíduos, incapacitando-os de reportar sinais aos profissionais de saúde 24,25. Todos estes dados reportados pela literatura são de grande interesse na implementação de estratégias de notificação de reações adversas a medicamentos pela UFPorto.

A taxa de notificação, como um dos indicadores de desempenho da UFPorto, é bastante influenciada pelas estratégias implementadas para a promoção de reações adversas a medicamentos. No entanto o número de notificações anuais deve ser visto à luz do contexto geográfico e das respectivas estratégias. No Brasil, embora com maior número de notificações anuais comparativamente com Portugal, verifica-se um número inferior de sinais de segurança e, consequentemente, menor taxa de notificação de suspeita de reações adversas a medicamentos 26 . Esse facto alerta para a importância da qualidade da informação notificada, podendo esses dados dificultar a geração de sinais de segurança. Apesar de a notificação espontânea ser o método que mais contribui para a busca de dados na atividade de rotina em farmacovigilância, a subnotificação é um problema que potencia uma subestimação 
da frequência de reações adversas a medicamentos. Nesse sentido, importa recorrer a outros métodos conhecidos de grande utilidade (farmacovigilância ativa), complementando a informação recolhida pela notificação espontânea 15,27,28,29. Também a implementação de Estudos de Segurança Pós--autorização (PASS) poderá constituir um método de verificação de hipóteses. Os estudos observacionais, com diferentes desenhos de estudos (e.g.: caso-controle, coorte), contribuindo para o estudo da janela de segurança dos medicamentos em contexto real e, por isso, não sujeitos a critérios de inclusão de participantes tão restritos como nos ensaios clínicos.

À semelhança do que é reportado na literatura, a integração dos sistemas de informação dos serviços de saúde nas bases de dados de farmacovigilância permite recolher informação detalhada acerca da utilização dos medicamentos e suspeitas de reações adversas a medicamentos que, de outra forma, não seriam reportados ao SNF 30 . Uma vez que esta integração de dados envolve o registo clínico dos doentes, tem de se criar condições de acesso à informação clínica, atendendo ao Regulamento Geral sobre a Proteção de Dados. Neste sentido, em 2011 foi desenvolvido um estudo com o objetivo de comparar o potencial das bases de dados administrativas hospitalares na detecção de reações adversas a medicamentos e qual poderia ser o seu contributo para o SNF 31. Para o efeito, a UFPorto coordenou um estudo retrospetivo no Centro Hospitalar e Universitário de São João, no Porto, em que se compararam as reações adversas a medicamentos detectadas pela base de dados clínico administrativa hospitalar com as que tinham sido notificadas ao SNF provenientes do mesmo hospital, no mesmo período. Daqui, concluiu-se que a base de dados clínica administrativa hospitalar tem potencial como ferramenta de informação secundária à base de dados de farmacovigilância - Portal RAM - do Infarmed. Idealmente, a integração de bases de dados deveria funcionar como um sistema completo de apoio à decisão clínica e de alerta para suspeitas de reações adversas a medicamentos 30 . O SIRAI (Sistema de Informação de Reações Adversas e Incidentes) foi implementado pela UFPorto em 2018 na Unidade Local de Saúde de Matosinhos, também no distrito do Porto, como uma base de dados única que reúne todas as reações adversas a medicamentos detectadas na instituição.

No que concerne às instituições notificadoras, os hospitais são aqueles que mais notificam, estando em linha de conta com o que já foi anteriormente descrito na literatura 19,20,32. Estes resultados remetem para a importância dos profissionais de saúde na detecção de reações adversas a medicamentos em doentes hospitalizados, dada a proximidade entre ambos durante o período hospitalização. No caso dos centros de saúde e das farmácias comunitárias, estando limitadas ao autorrelato do usuário, assumem esta condição como uma condicionante na detecção, identificação e posterior notificação da reações adversas a medicamentos. Também a falta de tempo e a dificuldade na identificação do medicamento suspeito são barreiras descritas na literatura 33. Por outro lado, o farmacêutico, enquanto profissional de saúde, é aquele que mais notifica suspeitas de reações adversas a medicamentos depois do médico, estando em linha com os resultados descritos em publicações oficiais 4,16. Embora não tenhamos dados sobre a distribuição dos farmacêuticos por serviços de saúde, a literatura mostra que os farmacêuticos hospitalares notificam mais que os farmacêuticos comunitários 34 . Este dado pode ainda estar associado ao fato de os profissionais notificarem sobretudo reações adversas classificados como "graves", e estas tendem a acontecer principalmente em doentes hospitalizados 32 . Pouco esperadas são as taxas de subnotificação assumidas pelos enfermeiros, possivelmente associadas ao facto de reportarem diretamente ao médico ou ao farmacêutico hospitalar. Os enfermeiros são responsáveis pela administração e monitorização da terapêutica, tornando-se assim elementos-chave para identificar iatrogenia medicamentosa 35 .

Ainda relativamente à distribuição anual, verifica-se um pico no ano de $2004(\mathrm{n}=640)$ comparando-se aos anos adjacentes, motivado por uma grande intervenção educativa nos profissionais de saúde. $\mathrm{O}$ estudo em causa envolveu intervenções educativas para médicos e farmacêuticos, e que fossem de encontro às razões apontadas para as altas taxas de subnotificação 36,37. A literatura tem reportado que uma das principais razões mencionadas pelos profissionais de saúde para não notificarem as suspeitas de reações adversas a medicamentos está relacionada com o aumento do volume de trabalho 38,39, pelo que as intervenções educativas têm de ser o mais incisivas possíveis. Da análise destas intervenções, verificou-se um forte contributo para o aumento das notificações de reações adversas a medicamentos, embora tenham se mostrado ineficazes com o decorrer do tempo 36,37. Assim, em 2007 foi efetuado um estudo de reforço que incluiu as entrevistas telefônicas às intervenções educativas já implementadas anteriormente 40,41. As conclusões do estudo dão conta que as intervenções tele- 
fônicas contribuíram para o aumento da notificação de reações adversas a medicamentos até quatro meses após a intervenção, enquanto que as intervenções educativas se prolongaram até um ano 40. Já em 2009, foi realizado um estudo de intervenção que se baseava na inclusão de hyperlinks diretos para o formulário de notificação online nos registos clínicos eletrônicos e/ou nos ambientes de trabalho dos profissionais de saúde de hospitais da região do Porto, reportando aumentos significativos da notificação das reações adversas a medicamentos mais relevantes (graves e inesperadas) 42. Em 2016, foi publicado um estudo comparativo de todas as estratégias de promoção da notificação espontânea de reações adversas a medicamentos implementadas até a data na UFPorto. Todas as estratégias estudadas aumentaram o número de notificações de reações adversas a medicamentos, tendo-se verificado que o maior aumento deveu-se a protocolos estabelecidos com departamentos médicos a nível hospitalar ${ }^{43}$. Assim, e no que diz respeito ao contributo para a investigação científica, a UFPorto tem desenvolvido diversos trabalhos no âmbito da farmacovigilância e da farmacoepidemiologia, contando já com 27 artigos publicados em revistas científicas com mais de 300 citações. A Unidade está também associada a vários projetos de investigação na segurança do medicamento, como é o caso do inquérito internacional sobre retinoides orais e valproatos, com o objetivo de avaliar a utilização deste medicamento na União Europeia.

Mais recentemente, a UFPorto associou-se a um consórcio Europeu para monitorização da segurança das vacinas contra a COVID-19, estando a colaborar com outras duas unidades de farmacovigilância portuguesas na monitorização de populações especiais em Portugal.

Como estratégia de cooperação direta com as instituições de saúde, a UFPorto é também constituída pelos delegados de farmacovigilância, que têm colaborado de forma muito consistente ao longo dos anos de atividade da unidade. Os delegados de farmacovigilância são profissionais de saúde que, nas instituições de saúde onde exercem sua atividade profissional, divulgam o sistema de farmacovigilância e promovem de forma ativa e de grande proximidade a notificação espontânea de reações adversas a medicamentos juntos dos restantes profissionais de saúde. Atualmente, a equipe de delegados é constituída por 11 elementos, distribuídos por sete instituições de saúde do distrito do Porto.

Paralelamente, a UFPorto assenta sua atividade anual em múltiplas ações de formação na promoção da atividade em farmacovigilância nas suas múltiplas vertentes e, em particular, na capacitação dos usuários, estudantes e profissionais de saúde para a notificação de suspeitas de reações adversas a medicamentos. Estas ações de formação decorrem habitualmente em instituições de saúde e outras de âmbito acadêmico e social.

\section{Perspectivas futuras}

A análise descritiva do nosso estudo permitiu concluir que os profissionais de saúde e os usuários do Norte de Portugal, nomeadamente do distrito do Porto, têm vindo a contribuir significativamente para o ecossistema nacional da segurança do medicamento.

Este trabalho retrospectivo identificou variabilidade no número de notificações ao longo dos anos, fortemente influenciada pelas diferentes iniciativas implementadas. A experiência da UFPorto mostra que estas iniciativas, embora úteis, tendem a perder importância junto dos notificadores (profissionais de saúde e usuários). As ações formativas periódicas continuam a ser as iniciativas que mais impacto têm junto do notificador, sobretudo com a descentralização desde 2017 e com o aumento do número de unidades regionais. A implementação de estratégias singulares tende a não ter um impacto tão significativo como seria de esperar, pelo que se reforça a importância da manutenção de várias iniciativas e sistemas de informação em simultâneo e, se possível, devidamente integradas.

Ao longo de aproximadamente vinte anos de atividade da UFPorto enquanto unidade regional, verificou-se um aumento da taxa de notificação global e, em particular, de notificação de reações adversas graves a medicamentos e não descritas no RCM, evidenciando maior consciência por parte dos notificadores das implicações da farmacovigilância na avaliação da segurança dos medicamentos. As variações globais observadas ao longo dos anos são resultado da alteração do paradigma da farmacovigilância nas últimas duas décadas. Foi observada uma mudança na consciência da farmacovigilância como uma simples atividade de recolha e tratamento de suspeitas de reações adversas a medicamentos, para uma abordagem mais complexa e proativa na gestão do risco. O SNF de Portugal, 
à semelhança de outros sistemas nacionais e internacionais, deverá incorporar as tendências de evolução, no sentido de simplificar e racionalizar os seus processos, sempre que possível, de forma a minimizar a carga regulamentar para todas as partes interessadas. A título de exemplo, em 2019 a UFPorto, no âmbito da sua atividade na gestão de sinais de risco, detectou aumento de queixas relativamente ao medicamento goserrelina teva (implante subcutâneo de 3,6mg). As notificações reportavam dor intensa sentida durante a administração e dificuldade do ato de administração do medicamento por parte do enfermeiro. Apesar de se tratarem de reações adversas a medicamentos aparentemente não graves e descritas no RCM, a UFPorto entendeu classificar essas reações adversas a medicamentos como graves, uma vez que inibiam as usuários de administrações posteriores, constituindo um grave problema de adesão à terapêutica. A dificuldade na administração da goserrelina deste laboratório em particular parecia relacionar-se com a agulha romba na ponta e com o sistema de recolha lento (rudimentar) da agulha após a administração do medicamento, o que tornava a administração extremamente dolorosa. Com base nas 36 notificações recebidas pela UFPorto, o Infarmed entendeu a suspensão deste medicamento em específico.

O futuro dos sistemas de farmacovigilância passa por uma aposta assente em três pilares fundamentais. Em primeiro lugar, a implementação de novas estratégias de busca, processamento e armazenamento de dados, nas quais se incluem o Big Data, a Real World Data, os registos clínicos e as redes sociais. Em segundo, o desenvolvimento e aplicação de novas soluções tecnológicas, com a implementação de novos webservices para extração de informação de diversas bases de dados ou no apoio à monitorização e detecção de erros de medicação (e.g.: erros de utilização de medicamentos loolalike sound-alike). Por fim, e como elo de todas as abordagens, as pessoas. A participação dos doentes como fonte única de informação na utilização de medicamentos é essencial, contribuindo para uma maior credibilização desta participação junto dos profissionais de saúde, das agências reguladoras e dos decisores. Destacamos o apoio crescente da informática biomédica no desenvolvimento de grande parte das infraestruturas tecnológicas em farmacovigilância 28,44. Estes contributos tecnológicos na área da saúde (e-Health) têm sido integrados gradualmente em todo o ciclo de farmacovigilância 28,45. A informática biomédica tem também permitido desenvolver métodos analíticos mais robustos, tais como os métodos de análise da desproporcionalidade. Esta classe de métodos analíticos permite uma análise dos dados resultantes da notificação espontânea de reações adversas a medicamentos, por se concentrarem em várias medidas estatísticas de associação 46. Além disso, o recurso à inteligência artificial como método de apoio à decisão em atividades de farmacovigilância tem se mostrado muito útil, por exemplo, como suporte a atividades de imputação de causalidade (e.g.: Bayesian Network Approach) 47.

Desde 1963, a farmacovigilância foi reconhecida como uma área prioritária na saúde pública a nível global pela OMS, garantindo a monitorização permanente da janela de segurança dos medicamentos introduzidos no mercado. Mais tarde, em 1992, também Portugal avançou com a criação do SNF, tendo desde logo começado a contribuir para a recolha e avaliação sistemática de reações adversas a medicamentos. O carácter descentralizado - por meio das unidades regionais de farmacovigilância - e de notificação obrigatória assumido pelo SNF, tem contribuído para uma vigilância ativa, próxima das instituições de saúde e com papel muito relevante na capacitação dos profissionais de saúde e dos usuários na promoção da notificação espontânea de reações adversas a medicamentos. Também a atividade acadêmica e científica nas unidades regionais de farmacovigilância têm contribuído de forma muito significativa para alimentar o ecossistema da farmacovigilância e da farmacoepidemiologia, estudando novas intervenções para a promoção da notificação espontânea, estudo de avaliação de padrões de risco de ocorrência de reações adversas a medicamentos e no reporte científico de reações adversas. Torna-se, assim, indiscutível o papel relevante que a farmacovigilância tem assumido ao longo dos anos, seja a nível da indústria farmacêutica (pelos titulares de AIM) ou pela entidade reguladora do medicamento - o Infarmed, tendo como premissa a segurança do doente. 


\section{Colaboradores}

Todos os autores contribuíram igualmente para a elaboração deste artigo.

\section{Informações adicionais}

ORCID: Renato Ferreira-da-Silva (0000-00016517-6021); Inês Ribeiro-Vaz (0000-0002-34428158); Ana Marta Silva (0000-0003-2355-2310); Joana Marques (0000-0003-1043-5078); Jorge Junqueira Polónia (0000-0002-0133-7217).

\section{Agradecimentos}

Este trabalho foi financiado por Fundos Nacionais e comunitários do Fundo Social Europeu (FSE) através da FCT - Fundação para a Ciência e a Tecnologia (Portugal), no âmbito da bolsa de doutoramento 2020.10231.BD (DOCTORATES 4 COVID-19) de Renato Ferreira-da-Silva.

\section{Referências}

1. Committee on Safety of Drugs. Report for 1969 and 1970. London: Her Majesty's Stationery Office; 1971.

2. World Health Organization. The importance of pharmacovigilance. Geneva: World Health Organization; 2002.

3. Parlamento Europeu; Conselho da União Europeia. Diretiva 2010/84/EU do Parlamento Europeu e do Conselho de 15 de Dezembro. Estrasburgo: Conselho da União Europeia; 2010.

4. Autoridade Nacional do Medicamento e Produtos de Saúde. Farmacovigilância em Portugal: 25 anos: INFARMED. http://app10.infar med.pt/e_book_farmacovigilancia25/index. html (acessado em 02/Jun/2020).

5. Wax PM. Elixirs, diluents, and the passage of the 1938 Federal Food, Drug and Cosmetic Act. Ann Intern Med 1995; 122:456-61.

6. National Archives and Records Administration. Act of June 25, 1938 (Federal Food, Drug, and Cosmetic Act), Public Law 75-717, 52 STAT 1040, which prohibited the movement in interstate commerce of adulterated and misbranded food, drugs, devices, and cosmetics. https://catalog.archives.gov/id/299847 (acessado em 02/Jun/2020).

7. Isenberg SJ. The fall and rise of chloramphenicol. J AAPOS 2003; 7:307-8.

8. Lokhande J, Juvekar AS, Kulkarni KP. Chloramphenicol: screening and review to evaluate its potential beneficial effects in leukaemia. J Indian Med Assoc 2007; 105:224, 226-8.

9. McBride WG. Thalidomide and congenital abnormalities. Lancet 1961; 278:1358.

10. Autoridade Nacional do Medicamento e Produtos de Saúde. Resumo das características do medicamento: talidomida. https://extranet.in farmed.pt/INFOMED-fo/pesquisa-avancada. xhtml (acessado em 02/Jun/2020).

11. Feldschreiber P, Breckenridge A. After thalidomide - do we have the right balance between public health and intellectual property. Rev Recent Clin Trials 2015; 10:15-8.

12. Uppsala Monitoring Centre. The use of the WHO-UMC system for standardised case causality assessment. https://www.who-umc. org/media/164200/who-umc-causalityassessment_new-logo.pdf (acessado em 30/ Ago/2021).

13. European Medicines Agency. ICH E2A Clinical safety data management: definitions and standards for expedited reporting. https:// www.ema.europa.eu/en/ich-e2a-clinicalsafety-data-management-definitions-stan dards-expedited-reporting (acessado em 04/ Jun/2020)

14. Karch FE, Lasagna L. Toward the operational identification of adverse drug reactions. Clin Pharmacol Ther 1977; 21:247-54.

15. Noren GN, Edwards IR. Modern methods of pharmacovigilance: detecting adverse effects of drugs. Clin Med (Lond) 2009; 9:486-9. 
16. Autoridade Nacional do Medicamento e Produtos de Saúde. Notificação de reações adversas a medicamentos: desempenho do Sistema Nacional de Farmacovigilância desde 2012. https://www.infarmed.pt/web/infarmed/en tidades/medicamentos-uso-humano/farmaco vigilancia/desempenho-do-snf? fbclid = IwAR 2newrgP1U44sihYWC4a-PgqmI35xNuIDG v7dHdLqTo4WKV9vzqL0t4Pag (acessado em 04/Jun/2020).

17. Rolfes L, Wilkes S, van Hunsel F, van Puijenbroek E, van Grootheest K. Important information regarding reporting of adverse drug reactions: a qualitative study. Int J Pharm Pract 2014; 22:231-3.

18. Batel-Marques F, Mendes D, Alves C, Penedones A, Dias P, Martins A, et al. Farmacovigilância em Portugal: atividade da unidade regional do centro. Acta Med Port 2015; 28:222-32.

19. Marques J, Ribeiro-Vaz I, Pereira AC, Polonia J. A survey of spontaneous reporting of adverse drug reactions in 10 years of activity in a pharmacovigilance centre in Portugal. Int $\mathrm{J}$ Pharm Pract 2014; 22:275-82.

20. Lazarou J, Pomeranz BH, Corey PN. Incidence of adverse drug reactions in hospitalized patients: a meta-analysis of prospective studies. JAMA 1998; 279:1200-5.

21. European Commission. Proposal for a regulation amending, as regards pharmacovigilance of medicinal products for human use. Regulation (EC) No 726/2004. Impact assessment; 2008. https://ec.europa.eu/health// sites/health/files/files/pharmacos/pharm pack_12_2008/pharmacovigilance-ia-vol1_ en.pdf (acessado em 06/Jun/2020).

22. Khalil $\mathrm{H}$, Huang $\mathrm{C}$. Adverse drug reactions in primary care: a scoping review. BMC Health Serv Res 2020; 20:5.

23. Masotti P, McColl MA, Green M. Adverse events experienced by homecare patients: a scoping review of the literature. Int J Qual Health Care 2010; 22:115-25.

24. Onder G, Gambassi G, Scales CJ, Cesari M, Vedova CD, Landi F, et al. Adverse drug reactions and cognitive function among hospitalized older adults. Eur J Clin Pharmacol 2002; 58:371-7.

25. Kanagaratnam L, Drame M, Trenque T, Oubaya N, Nazeyrollas P, Novella JL, et al. Adverse drug reactions in elderly patients with cognitive disorders: a systematic review. Maturitas 2016; 85:56-63.

26. Pepe VLE, Novaes HMD. Sistema Nacional de Farmacovigilância no Brasil e em Portugal: semelhanças, diferenças e desafios. Cad Saúde Pública 2020; 36:e0043019.

27. Layton D, Hazell L, Shakir SA. Modified prescription-event monitoring studies: a tool for pharmacovigilance and risk management. Drug Saf 2011; 34:e1-9.

28. Noren GN. Pharmacovigilance for a revolving world: prospects of patient-generated data on the internet. Drug Saf 2014; 37:761-4.
29. Mann RD, Andrews EB. Data mining in pharmacovigilance: a view from the uppsala monitoring centre. In: Mann RD, Andrews EB, editores. Pharmacovigilance. 2nd Ed. New York: John Wiley \& Sons; 2007. p. 265-76.

30. Cruz-Cunha MM, Miranda IM. Encyclopedia of e-health and telemedicine. Hershey: Idea Group; 2016.

31. Marques J, Herdeiro MT, Freitas A, Silva AM, Costa-Pereira A. Strategies for detection adverse drug reactions-comparison of hospital database and spontaneous reporting. Drug Saf 2015; 38:952.

32. Giardina C, Cutroneo PM, Mocciaro E, Russo GT, Mandraffino G, Basile G, et al. Adverse drug reactions in hospitalized patients: results of the FORWARD (Facilitation of Reporting in Hospital Ward) Study. Front Pharmacol 2018; 9:350.

33. Hughes ML, Weiss M. Adverse drug reaction reporting by community pharmacists - the barriers and facilitators. Pharmacoepidemiol Drug Saf 2019; 28:1552-9.

34. van Grootheest AC, Jong-van den Berg LTW. The role of hospital and community pharmacists in pharmacovigilance. Res Social Adm Pharm 2005; 1:126-33.

35. Mendes D, Alves C, Batel Marques F. Nurses' spontaneous reporting of adverse drug reactions: expert review of routine reports. J Nurs Manag 2014; 22:322-30.

36. Herdeiro MT, Figueiras A, Polonia J, GestalOtero JJ. Influence of pharmacists' attitudes on adverse drug reaction reporting: a casecontrol study in Portugal. Drug Saf 2006; 29:331-40.

37. Herdeiro MT, Figueiras A, Polonia J, GestalOtero JJ. Physicians' attitudes and adverse drug reaction reporting: a case-control study in Portugal. Drug Saf 2005; 28:825-33.

38. Lee SB, Schepers GP, Goldberg KL. Electronic adverse-drug-reaction-reporting program. Am J Health Syst Pharm 2004; 61:1230.

39. Hinrichsen VL, Kruskal B, O’Brien MA, Lieu TA, Platt R. Using electronic medical records to enhance detection and reporting of vaccine adverse events. J Am Med Inform Assoc 2007; 14:731-5.

40. Herdeiro MT, Ribeiro-Vaz I, Ferreira M, Polonia J, Falcao A, Figueiras A. Workshop- and telephone-based interventions to improve adverse drug reaction reporting: a clusterrandomized trial in Portugal. Drug Saf 2012; 35:655-65.

41. Ribeiro-Vaz I, Herdeiro MT, Polónia J, Figueiras A. Estratégias para aumentar a sensibilidade da farmacovigilância em Portugal. Rev Saúde Pública 2010; 45:129-35.

42. Ribeiro-Vaz I, Santos C, Costa-Pereira A, Cruz-Correia R. Promoting spontaneous adverse drug reaction reporting in hospitals using a hyperlink to the online reporting form: an ecological study in Portugal. Drug Saf 2012; 35:387-94. 
43. Ribeiro-Vaz I, Santos CC, Cruz-Correia R. Promoting adverse drug reaction reporting: comparison of different approaches. Rev Saúde Pública 2016; 50:14.

44. U.S. Food \& Drug Admninistration. FDA Adverse Event Reporting System (FAERS) electronic submissions. https://www.fda.gov/ drugs/questions-and-answers-fdas-adverseevent-reporting-system-faers/fda-adverseevent-reporting-system-faers-electronic-sub missions (acessado em 12/Jun/2020).

45. Rudin RS, Bates DW, MacRae C. Accelerating innovation in health IT. N Engl J Med 2016; 375:815-7.
46. Harpaz R, Vilar S, Dumouchel W, Salmasian $\mathrm{H}$, Haerian K, Shah NH, et al. Combing signals from spontaneous reports and electronic health records for detection of adverse drug reactions. J Am Med Inform Assoc 2013; 20:413-9.

47. Rodrigues PP, Ferreira-Santos D, Silva A, Polonia J, Ribeiro-Vaz I. Causality assessment of adverse drug reaction reports using an expertdefined Bayesian network. Artif Intell Med 2018; 91:12-22. 
Abstract

Since 1963, the World Health Organization has acknowledged pharmacovigilance as a priority area in global public health, guaranteeing permanent monitoring of drug safety. This study aimed to characterize the reports of adverse drug reactions received by the Porto Pharmacovigilance Centre (UFPorto), Portugal, in the unit's two decades of work. The analysis included all reports of suspected adverse drug reactions received from January 2001 to December 2019. We calculated the annual reporting rates and distribution by origin, type of notifier and place of work, severity, prior knowledge, and causality of the reported adverse drug reactions. During the study period, UFPorto received 9,711 notifications of suspected adverse drug reactions. Hospital institutions reported the most suspected adverse drug reactions $(n=6,003 ; 64 \%)$, as did physicians among healthcare workers $(n=5,284 ; 54.4 \%)$. The most frequently reported adverse events were severe $(n=6,275 ; 72 \%)$ and are described in the respective Summary of Product Characteristics $(n=$ 6,978; 72\%). Most of the reports received by UFPorto were evaluated as having "probable" causality $(n=7,473 ; 77 \%)$, independently of the type of notifier. The results are consistent with other data previously reported in the international medical literature and official national reports. However, the underreporting rates are still higher than expected. In approximately 20 years, UFPorto has experienced an increase in its activity in various areas of drug safety.

Pharmacovigilance; Drug-Related Side Effects and Adverse Reactions; Adverse Drug Reaction Reporting System; Drug Monitoring

\section{Resumen}

Desde 1963 la farmacovigilancia fue reconocida por la Organización Mundial de la Salud, como un área prioritaria en la salud pública a nivel global, garantizando la monitorización permanente de la seguridad de los medicamentos. El objetivo de este trabajo fue caracterizar las reacciones adversas a medicamentos recibidas por la Unidad de Farmacovigilancia de Oporto (UFPorto), Portugal, a lo largo de dos décadas de actividad. Se consideraron todas las notificaciones de sospechas de reacciones adversas a medicamentos, recibidas entre enero de 2001 y diciembre de 2019. Se calcularon las tasas de notificación anuales, así como su distribución por origen, tipo de notificador y lugar de ejercicio de la actividad, gravedad, conocimiento previo y causalidad de las reacciones adversas a medicamentos notificadas. En el período de estudio, la UFPorto recibió 9.711 notificaciones de sospechas reacciones adversas a medicamentos. Las instituciones hospitalarias son quienes más notifican $(n=6.003 ; 64 \%)$, así como el médico entre los profesionales de salud $(n=5.284 ; 54,4 \%)$. Los eventos adversos más frecuentemente reportados son graves $(n=6.275 ; 72 \%)$ y se encuentran descritos en lo respectivo Resumen de las Características del Medicamento $(n=6978 ; 72 \%)$. A la mayoría de las notificaciones evaluadas por la UFPorto se le atribuyó el grado de causalidad "probable" ( $n=7.473 ; 77 \%)$, independientemente del tipo de notificador. Los resultados obtenidos son concordantes con otros datos previamente reportados en la literatura médica internacional y en informes oficiales nacionales. No obstante, se continúa verificando tasas de subregistro acentuadas, frente a lo esperado. A lo largo de aproximadamente 20 años de actividad de la UFPorto, se ha verificado un aumento de su actividad en las diversas vertientes de la seguridad del medicamento.

Farmacovigilancia; Efectos Colaterales

$y$ Reacciones Adversas Relacionados con

Medicamentos; Sistemas de Registro de Reacción

Adversa a Medicamentos; Monitoreo de Drogas
Recebido em 23/Out/2020

Versão final reapresentada em 14/Jan/2021

Aprovado em 12/Fev/2021 